\title{
Identification of an Endoplasmic Reticulum-Retention Motif in an Intracellular Loop of the Kainate Receptor Subunit KA2
}

\author{
Yukiko Nasu-Nishimura, ${ }^{1}$ David Hurtado, ${ }^{1}$ Stephanie Braud, ${ }^{1,2}$ Tina Tze-Tsang Tang, ${ }^{1}$ John T. R. Isaac, ${ }^{1,2}$ and \\ Katherine W. Roche ${ }^{1}$ \\ ${ }^{1}$ National Institute of Neurological Disorders and Stroke, National Institutes of Health, Bethesda, Maryland 20892, and ${ }^{2}$ Medical Research Council Centre \\ for Synaptic Plasticity, Department of Anatomy, University of Bristol, Bristol BS8 1TD, United Kingdom
}

Neuronal kainate receptors are typically heteromeric complexes composed of GluR5-7 and KA1-2 subunits. Although GluR5-7 can exist as functional homomeric channels, the KA subunits cannot. KA2 is widely expressed in the CNS, and KA2/GluR6 heteromers are the most prevalent subunit composition in brain. Previous work has identified endoplasmic reticulum (ER)-retention motifs in the $\mathrm{C}$ terminus of KA2, which prevent surface expression of KA2 homomers. However, we find that, when these motifs are mutated, only a small fraction of KA2 is surface expressed. We now identify an additional ER retention motif in the intracellular loop region of KA2, which, when mutated together with the C-terminal motifs, significantly increases the level of KA2 surface expression. However, electrophysiological analysis of surface-expressed KA2 homomers indicates that they do not form functional ion channels. In heterologous cells, a large fraction of KA2 remains intracellular even when the trafficking motifs are mutated or when GluR6 is coexpressed. Therefore, we analyzed the trafficking of endogenous KA2 in vivo. We find that native KA2 surface expression is dramatically reduced in GluR6 knock-out mice compared with wild-type mice. In contrast, KA2 trafficking was unaffected in the GluR5 knock-out. Thus, our study demonstrates that trafficking motifs in both the intracellular loop and C terminus regulate KA2 surface expression; however, in neurons, GluR6 oligomerization is required for egress of KA2 from the ER and transport to the cell surface. The combination of these mechanisms likely prevents surface expression of nonfunctional KA2 homomers and ensures a high level of GluR6/KA2 heteromeric kainate receptors.

Key words: GluR6; glutamate receptor; trafficking; ER-retention; oligomerization; surface expression

\section{Introduction}

Kainate receptors are widely distributed throughout the brain at both presynaptic and postsynaptic sites (Lerma, 2003; Isaac et al., 2004), where they modulate neurotransmitter release or mediate excitatory neurotransmission, respectively. Kainate receptors exist as both homomeric and heteromeric complexes, with subunit composition affecting the pharmacological and physiological properties of the receptors. The kainate receptor subunits (GluR5-7, KA1-2) have distinct, yet overlapping, distributions in the CNS (Wisden and Seeburg, 1993; Bahn et al., 1994; Porter et al., 1997). The most prevalent endogenous kainate receptors are thought to be heteromers containing the GluR6 and KA2 subunits (Lerma, 2003). Unlike GluR6, KA2 does not exist as functional homomers, and it is likely that neurons use precise quality control mechanisms to regulate the oligomerization and trafficking of KA2. Recently, it was demonstrated that KA2 is

Received Feb. 8, 2006; revised May 17, 2006; accepted May 18, 2006.

This work was supported by the National Institute of Neurological Disorders and Stroke (NINDS) Intramural Research Program and the Wellcome Trust (J.T.R.I.). We thank Dr. D. Maric (NINDS FACS Facility) for advice and expertise in the analysis of FACS data and Dr. C. L. Smith (NINDS Light Imaging Facility) for assistance with confocal microscopy. In addition, we acknowledge J. Nagle and D. Kauffman (NINDS Sequencing Facility) for DNA sequencing We also thank Dr. S. F. Heinemann for providing GluR5 KO mice and GluR6 KO mice and Dr. M. Gasior for assistance in maintaining those mice.

Correspondence should be addressed to Katherine W. Roche, National Institute of Neurological Disorders and Stroke, National Institutes of Health, Building 35, Room 2C903, Bethesda, MD20892.E-mail: rochek@ninds.nih.gov. D01:10.1523/JNEUROSCI.0573-06.2006

Copyright $\odot 2006$ Society for Neuroscience $\quad$ 0270-6474/06/267014-08\$15.00/0 endoplasmic reticulum (ER) retained when expressed in heterologous cells (Gallyas et al., 2003; Hayes et al., 2003; Ren et al., 2003). The molecular mechanisms regulating KA2 ER-retention likely play a critical role in regulating the complement of synaptic kainate receptors, because they ensure that KA2 only reaches the plasma membrane as a heteromeric channel. Similar restraints are imposed on other glutamate receptor subunits, most notably the NR1 subunit, which is an essential component of all NMDA receptors, but is ER retained and only traffics to the plasma membrane as a heteromeric channel (Standley et al., 2000; Scott et al., 2001). Thus, ER-retention of various glutamate receptor subunits is of particular interest, and elucidating the mechanisms underlying subunit assembly and trafficking to synaptic sites may help reveal the physiological relevance of glutamate receptor assemblies in the brain.

Recently, Ren et al. (2003) reported the identification of an ER-retention motif and an endocytic motif within the $\mathrm{C}$ terminus of KA2. In the current study, we find that there is an additional ER-retention motif within a small intracellular loop of KA2. Mutation of this loop motif coupled with mutations of the previously identified C-terminal trafficking motifs significantly increases surface expression of KA2 in heterologous cells. However, even with the mutation of these ER-retention signals, the majority of $\mathrm{KA} 2$ is not surface expressed. For this reason, we also investigated the trafficking of endogenous KA2 in neurons by comparing KA2 surface expression in neurons from both wild-type and kainate 
receptor subunit knock-out $(\mathrm{KO})$ mice. We find that only a small fraction of KA2 is ER retained in brains from wild-type animals. Furthermore, GluR6 specifically regulates ER egress of KA2 in vivo, because the majority of KA2 is ER retained in GluR6 KO brain. In contrast, in the GluR5 $\mathrm{KO}, \mathrm{KA} 2$ surface expression is normal, indicating a specific role for oligomerization with GluR6 in the efficient trafficking of KA2 to the plasma membrane. Thus, we describe novel mechanisms regulating the surface expression of KA2, and demonstrate that regulation of KA2 expression at the neuronal plasma membrane is a complex process involving multiple KA2 ER-retention motifs and hetero-oligomerization with GluR6.

\section{Materials and Methods}

Cells. HeLa cells (American Type Culture Collection, Manassas, VA) were maintained in DMEM containing 10\% fetal bovine serum, $1 \%$ L-glutamine, and $0.1 \%$ gentamicin. Primary cortical neurons were prepared from postnatal day 0 (P0) to $\mathrm{P} 1$ mouse pups according to manufacturer's primary neuron culture protocol (Invitrogen, Carlsbad, CA) and grown in Neurobasal medium (Invitrogen) supplemented with glutamine and B-27 supplement (Invitrogen).

Constructs. KA2 and GluR6 in pRK5 mammalian expression vectors were obtained as gifts from Dr. P. Seeburg (Max Planck, Heidelberg, Germany), and Tac was from Dr. J. Bonifacino (National Institute of Child Health and Human Development-National Institutes of Health, Bethesda, MD). The FLAG epitope was inserted after the signal sequences of KA2 or GluR6 by site-directed mutagenesis according to the QuikChange protocol (Stratagene, La Jolla, CA). All mutations in KA2 ER-retention motifs were also engineered by site-directed mutagenesis according to the QuikChange protocol. The sequences of all mutants were confirmed by automated sequence analysis. For FACS analysis, the mutant constructs were inserted into an IRES-EGFP fragment at a SalI site after the KA2 stop codon. The orientation of the inserted fragment was confirmed by restriction enzyme analysis.

Antibodies. Anti-FLAG antibodies (mouse monoclonal and rabbit polyclonal) and anti- $\alpha$-tubulin antibody were purchased from Sigma (St. Louis, MO). Anti-KA2 antibody and anti-GluR6/7 antibody were purchased from Upstate (Lake Placid, NY). Anti-KDEL antibody was from Stressgen (San Diego, CA).

Immunostaining for colocalization analysis. HeLa cells grown on glass coverslips were transfected with the cDNAs indicated ( $4 \mu \mathrm{g}$ of DNA per well; six-well dish) as previously described (Hayes et al., 2003) and analyzed $36 \mathrm{~h}$ later. Transfected cells were washed once in PBS and fixed in $4 \%$ paraformaldehyde in PBS for $15 \mathrm{~min}$ at room temperature. The cells were then washed in PBS and permeabilized in $0.2 \%$ Triton $\mathrm{X}-100$ in PBS for $5 \mathrm{~min}$. The coverslips were incubated with rabbit anti-FLAG antibody or anti-KA2 antibody and anti-KDEL antibody for $1 \mathrm{~h}$ at room temperature. After multiple washes with PBS, the cells were incubated with Alexa 568 anti-mouse secondary antibody (1: 500; Invitrogen) and Alexa 488 anti-rabbit secondary antibody (1: 500 ) for $30 \mathrm{~min}$ at room temperature. The coverslips were washed and mounted with the ProLong Antifade kit (Invitrogen). The cells were visualized using a Zeiss LSM510 laser-scanning confocal microscope (Carl Zeiss, Thornwood, NY).

Immunostaining for surface expression. Transfected live HeLa cells grown on glass coverslips were prepared as described above. Transfected cells were washed once in ice-cold PBS and labeled with anti-FLAG M2 monoclonal antibody or anti-Tac antibody for 20 min on ice. After three washes in ice-cold PBS, the cells were labeled with Alexa 488 anti-mouse secondary antibodies (1:500) for $30 \mathrm{~min}$ on ice. The cells were again washed three times in ice-cold PBS and fixed in $4 \%$ paraformaldehyde in PBS for 15 min at room temperature and then permeabilized in $0.2 \%$ Triton X-100 in PBS for 5 min. The coverslips were incubated with anti-FLAG M2 monoclonal antibody or antiKA2 antibody for $30 \mathrm{~min}$ at room temperature. After multiple washes with PBS, the cells were incubated with Alexa 568 anti-mouse or anti-rabbit secondary antibody (1:500) for $30 \mathrm{~min}$ at room temperature. The coverslips were then washed and mounted with the ProLong
Antifade kit. The cells were visualized using a Zeiss LSM510 confocal microscope.

Glycosidase treatment and immunoblotting. Wild-type, GluR6 KO, and GluR5 KO mouse brains were sonicated in lysis buffer (TBS: 2 mM EDTA, $0.1 \mathrm{~mm}$ AEBSF [4-(2-aminoethyl)benzenesulfonyl fluoride], $1 \mu \mathrm{g} / \mathrm{ml}$ leupeptin, and $5 \mathrm{~mm}$ iodoacetamide) without detergent. After addition of Triton X-100 to a final concentration of $1 \%$, the lysates were incubated for $1 \mathrm{~h}$ at $4^{\circ} \mathrm{C}$, and centrifuged at 20,000 $\times g$ for $20 \mathrm{~min}$. Fifty micrograms of supernatant from each sample was treated in one of three ways: (1) untreated, (2) endoglycosidase Hf (endo H) (1500 U), or (3) PNGase $\mathrm{F}(750 \mathrm{U})$. All samples were incubated at $37^{\circ} \mathrm{C}$ for at least $4 \mathrm{~h}$, resolved by SDS-PAGE, transferred onto polyvinylidene difluoride (PVDF) membranes, and probed with either anti-KA2 $(1 \mu \mathrm{g} / \mathrm{ml})$ or antiGluR6/7 $(0.5 \mu \mathrm{g} / \mathrm{ml})$ antibodies. The blots were incubated with antirabbit HRP secondary antibodies (1:5000; Amersham Biosciences, Piscataway, NJ), followed by detection with ECL reagents (Pierce, Rockford, IL).

FACS analysis of cell surface protein. HeLa cells grown in $6 \mathrm{~cm}$ dishes were transiently transfected with FLAG-KA2-IRES-EGFP mutants as previously described (Hayes et al., 2003) or Lipofectamine 2000 (Invitrogen) according to the manufacturer's instructions. Transfected live HeLa cells were washed twice with ice-cold PBS containing 5 mM EDTA (PBS/ EDTA) and incubated with ice-cold PBS/EDTA for 10 min on ice. Detached $1 \times 10^{6}$ cells were transferred to polystyrene test tubes (VWR Scientific, South Plainfield, NJ) and labeled with anti-FLAG M2 monoclonal antibody $(10 \mu \mathrm{g}$ in $0.1 \mathrm{ml})$ for $30 \mathrm{~min}$ on ice. Cells were then washed three times with ice-cold PBS and incubated with phycoerythrinconjugated anti-mouse $\mathrm{IgG}_{1}$ secondary antibody ( $10 \mu \mathrm{g}$ in $0.1 \mathrm{ml}$; Caltag, Burlingame, CA) for $30 \mathrm{~min}$ on ice. Cells were washed three times and suspended in ice-cold PBS. The cells were immediately analyzed using a dual-laser FACSVantage SE flow cytometer (Becton Dickinson, Mountain View, CA). CellQuest Acquisition and Analysis software (Becton Dickinson) was used to acquire and quantify the fluorescence signal intensities and to graph the data as bivariate dot density plots. Fluorescence emissions of individual fluorophores were initially corrected for spectral overlap using "simple" single color controls and appropriate electronic compensation. The background fluorescence was determined using HeLa cells transfected with empty vector and stained as described above.

Biotinylation assay of cell surface protein. Transfected HeLa cells or primary cultured cortical neurons grown in $10 \mathrm{~cm}$ culture dishes were washed three times with ice-cold wash buffer (PBS containing $1 \mathrm{~mm}$ $\mathrm{MgCl}_{2}$ and $0.1 \mathrm{~mm} \mathrm{CaCl}$ ) and incubated with $0.25 \mathrm{mg} / \mathrm{ml} \mathrm{EZ-Link}$ Sulfo-NHS-LC-biotin (Pierce) in cold wash buffer for $20 \mathrm{~min}$ at $4^{\circ} \mathrm{C}$ with gentle agitation. Cells were washed immediately once with ice-cold quenching buffer ( $50 \mathrm{~mm}$ glycine in wash buffer) and washed three times in quenching buffer for $5 \mathrm{~min}$ each. The cells were lysed in lysis buffer [50 mm Tris-HCl, pH 7.5, 2 mм EDTA, 2\% Triton X-100, EDTA-free complete protease inhibitor (Roche, Indianapolis, IN)] and incubated for $30 \mathrm{~min}$ at $4^{\circ} \mathrm{C}$. The lysates were centrifuged at $20,000 \times g$ for $20 \mathrm{~min}$. One milligram of supernatant was then incubated with $60 \mu \mathrm{l}$ of $50 \%$ slurry of streptavidin-Sepharose beads (Pierce) for $2 \mathrm{~h}$ at $4^{\circ} \mathrm{C}$. Beads were pelleted by centrifugation, and supernatants were saved for the samples of intracellular protein pool. Beads were washed four times in lysis buffer and then washed two times in PBS. Biotinylated proteins were eluted and analyzed by immunoblotting as described above.

Electrophysiology. One to $2 \mathrm{~d}$ after plating, human embryonic kidney 293 (HEK-293) cells were transfected with plasmids $(0.8-1 \mu \mathrm{g})$ using either Superfect (Qiagen, Valencia, CA) according to the manufacturer's instructions or using the calcium phosphate coprecipitation method. Plasmids included an IRES-EGFP and transfected cells were identified by the GFP (green fluorescent protein) signal. Whole-cell patch-clamp recordings were made 2-3 d after transfection using electrodes (4-7 M $\Omega$ ) containing the following (in mM): $117 \mathrm{CsMeSO}_{4}, 8 \mathrm{NaCl}, 10 \mathrm{HEPES}, 4$ MgATP, and 0.3 NaGTP, pH 7.4, and 290 mOsm. The extracellular solution contained the following (in $\mathrm{mm}$ ): $130 \mathrm{NaCl}, 5 \mathrm{KCl}, 2 \mathrm{CaCl}_{2}, 25$ HEPES, 30 D-glucose, $\mathrm{pH}$ 7.3. Recordings were made in voltage-clamp mode using Axopatch 200-B (Molecular Devices, Sunnyvale, CA) with a 
holding potential of $-70 \mathrm{mV}$ and filtered at 5 $\mathrm{kHz}$. Series resistance and input resistance were monitored using a $-2 \mathrm{mV}$ step applied before each application. Data were digitized at $10 \mathrm{kHz}$, displayed, and analyzed on-line using pClamp 9 (Molecular Devices). Agonist was rapidly applied using a fast-flow theta tube perfusion system driven by a piezoelectric device (Burleigh Instruments, Victor, NY). Open tip 20-80\% exchange times were $<400 \mu$ s. For each cell, agonist was applied at least 10 times. For experiments with cells expressing KA2 or KA2 mutants, interleaved experiments with cells expressing either GluR6, GluR6/KA2, or GluR6/ KA2 mutants were performed. For each construct, at least five cells were tested from at least three different cultures.

\section{Results}

The KA2 kainate receptor subunit is widely expressed in the mammalian brain and forms functional heteromeric channels with GluR6, another widely distributed kainate receptor subunit. Recently, several groups reported that KA2 does not traffic to the cell surface when expressed alone in heterologous cells (Gallyas et al., 2003; Hayes et al., 2003; Ren et al., 2003). In addition, Ren et al. (2003) reported that KA2 contains both an ER-retention motif (862-RRRRR-866) and a putative endocytic motif (908-LL-909) within its C terminus (Fig. $1 A$ ) that regulate trafficking of the KA2 subunit and preclude the expression of homomeric KA2 receptors on the plasma membrane. Using the same approach as that of Ren et al. (2003), we made a chimeric protein combining Tac (the IL-2 receptor $\alpha$-chain) with the C terminus of KA2 (Tac-KA2) (Fig. 1B) and analyzed its surface expression. Cells expressing Tac-KA2 or Tac-KA2 $2_{\mathrm{CtermA} / \mathrm{B}}$ were labeled with anti-Tac antibody on ice for $20 \mathrm{~min}$ (green). The cells were fixed, permeabilized, and additional primary antibody was applied to label intracellular pools of receptor (red). Consistent with previous results, very little Tac-KA2 was detected on the cell surface. In contrast, Tac-KA2 $2_{\text {CtermA/B }}$, which contained mutations in the C-terminal trafficking motifs reported by Ren et al. (2003), was efficiently detected on the cell surface (Fig. $1 C)$. We then analyzed the intracellular distribution of Tac-KA2 $2_{\mathrm{CtermA} / \mathrm{B}}$ using double-labeling of KA2 with an anti-KDEL antibody, which recognizes endogenous ER-retained proteins and is commonly used as an ER marker. Tac-KA2 $2_{\mathrm{CtermA} / \mathrm{B}}$ was detected at the plasma membrane as well as in the ER, whereas Tac-KA2 was mostly colocalized with KDEL and not localized at the plasma membrane (Fig. $1 D)$. These results confirm the previous findings that the trafficking motifs (862-RRRRR-866 and 908-LL-909) in the C terminus of KA2 control the surface expression of Tac-KA2 (Ren et al., 2003).

However, because Ren et al. relied almost exclusively on the of $\mathrm{KA} 2_{\mathrm{CtermA} / \mathrm{B}}$ with KDEL.

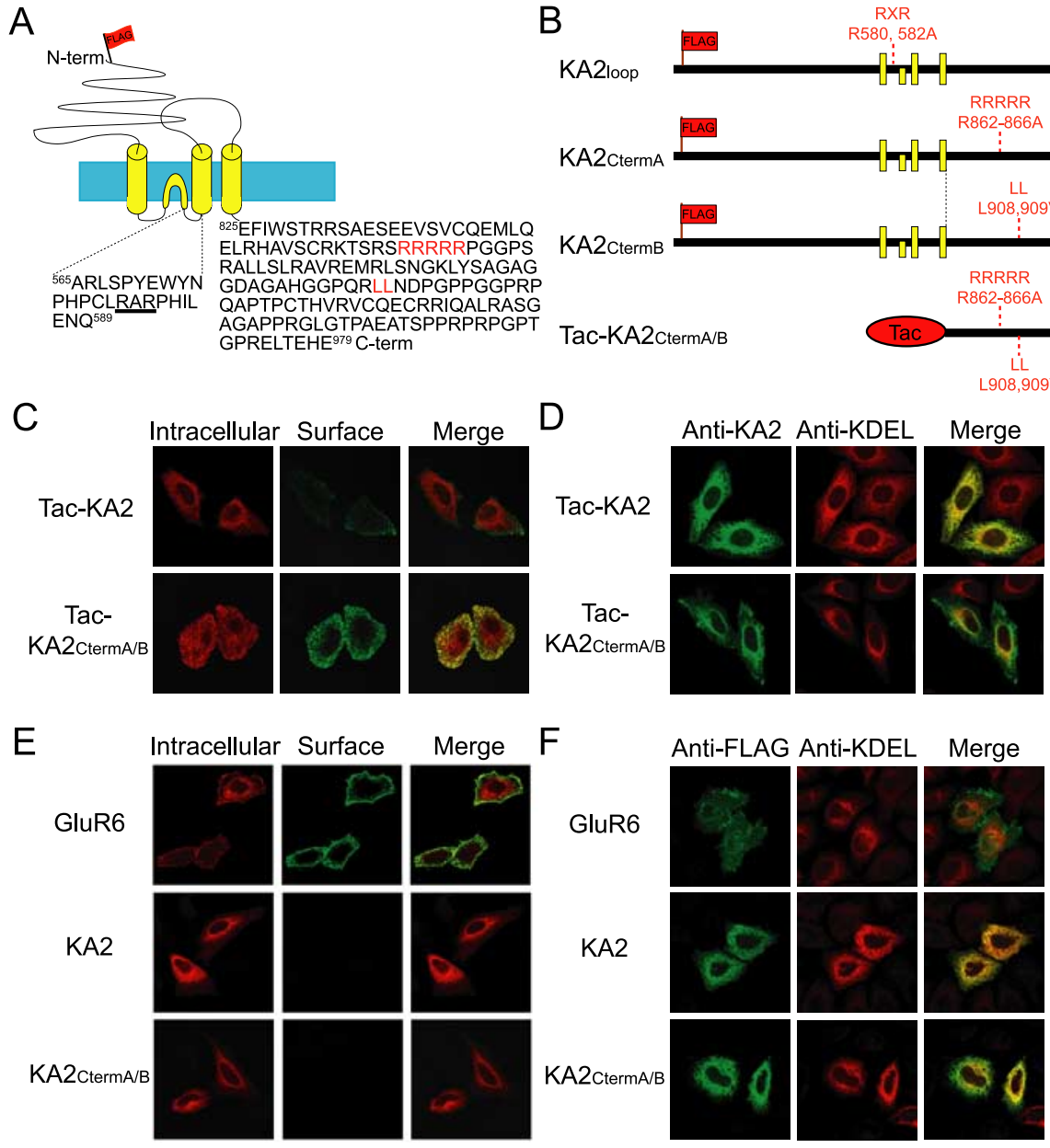

Figure 1. Mutations of C-terminal trafficking motifs do not allow robust surface expression of full-length KA2. $A$, Schematic diagram depicting the topology of KA2. The KA2 C terminus has 155 aa and contains several trafficking motifs that have been described previously: an arginine-rich motif (RRRRR) and a dileucine sequence (LL) are depicted in red. A consensus argininebased endocytic motif (RxR) within the intracellular loop is underlined. The FLAG epitope is depicted as a flag inserted just after the signal sequence in the $\mathrm{N}$ terminus of KA2. The yellow and blue boxes represent membrane domains and lipid bilayer, respectively. $\boldsymbol{B}$, Diagram illustrating mutations made in KA2. Tac-KA2 is a chimeric protein consisting of the plasma membrane protein Tac with the $C$ terminus of KA2. The yellow boxes represent membrane domains. Mutations in one or more of the trafficking motifs are indicated with a subscript representing the region of the mutation. $C, T a c-K A 2_{C t e r m A / B}$ is efficiently expressed on the plasma membrane. Live HeLa cells transfected with Tac-KA2 or Tac-KA2 ${ }_{\text {ctermA/B }}$ were labeled with anti-Tac antibody (surface) and Alexa 488-conjugated secondary antibody (green) on ice. The cells were fixed, permeabilized, and labeled with anti-Tac antibody (intracellular) and Alexa 568-conjugated secondary antibody (red). D, Tac-KA2 ${ }_{\mathrm{CtermA} / \mathrm{B}}$ is localized at the plasma membrane as well as the ER. HeLa cells transfected with Tac-KA2 or Tac-KA2 $2_{\text {(termA/B }}$ were fixed, permeabilized, and stained with antibodies against the (terminus of KA2 (green) and the ER marker KDEL (red). $E, K A 2_{C \text { termA/B }}$ is not expressed on the plasma membrane. Live HeLa cells transfected with FLAG-GluR6, FLAG-KA2, or FLAG-KA2 ${ }_{\text {(termA/B }}$ were labeled with anti-FLAG antibody (surface) and Alexa 488-conjugated secondary antibody (green) on ice. The cells were fixed, permeabilized, and labeled with anti-FLAG antibody (intracellular) and Alexa 568-conjugated secondary antibody (red). FLAG-GluR6 was used as a positive control for surfaceexpressed receptor. $\boldsymbol{F}$, Full-length $\mathrm{KA} 2$ is retained in the ER when expressed in HeLa cells, even with mutations in C-terminal

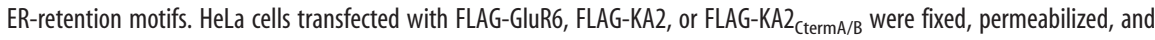
stained with antibodies against FLAG (green) and the ER marker KDEL (red). The merged images show the extensive colocalization

use of Tac as a plasma membrane reporter molecule as shown above, we extended our analyses to the trafficking and surface expression of the full-length KA2 subunit in heterologous cells and in neurons. We analyzed surface expression of KA2 and GluR6 using immunofluorescence miscroscopy. Surfaceexpressed receptors were labeled with anti-FLAG antibody on ice for $20 \mathrm{~min}$ (green). The cells were fixed, permeabilized, and additional primary antibody was applied to label intracellular pools of receptor (red). We found no striking difference between the 
surface-expression of wild-type $\mathrm{KA} 2$ and $\mathrm{KA} 2_{\mathrm{CtermA} / \mathrm{B}}$, which contained mutations of the C-terminal trafficking motifs, because both of these proteins displayed negligible expression on the plasma membrane (Fig. 1 E). As expected, GluR6 was robustly expressed on the plasma membrane when analyzed in parallel using the same staining assay (Fig. $1 E$ ). In a separate assay, we evaluated the intracellular distribution of full-length wild-type KA2, which is known to be ER retained (Gallyas et al., 2003; Hayes et al., 2003; Ren et al., 2003), KA2 ${ }_{\mathrm{CtermA} / \mathrm{B}}$ (Fig. 1 B), which has been reported to be surface expressed (Ren et al., 2003), and GluR6, which is efficiently expressed on the plasma membrane (Hayes et al., 2003; Ren et al., 2003). We transfected HeLa cells with full-length wild-type $\mathrm{KA} 2, \mathrm{KA} 2_{\mathrm{CtermA} / \mathrm{B}}$, or GluR6, and double-labeled cells with anti-FLAG antibody and anti-KDEL antibody. In sharp contrast to GluR6, we found that both wildtype KA2 and KA2 that contains mutations in the C-terminal trafficking motifs displayed extensive colocalization with KDEL (Fig. $1 F)$.

Based on our immunofluorescence microscopy data, we conclude that the mutations identified within the KA2 C terminus do not allow efficient surface expression of full-length KA2 on the plasma membrane of heterologous cells. Therefore, we began searching other regions of the KA2 sequence for putative ERretention motifs, and identified an RxR consensus ER-retention motif (Zerangue et al., 1999; Margeta-Mitrovic et al., 2000; Scott et al., 2001) within a small intracellular loop of KA2 (Fig. $1 A$ ). We mutated the RxR motif (R580,582A) and evaluated the surface expression of KA2 containing this mutation alone $\left(\mathrm{KA} 2_{\text {loop }}\right)$ or together with the C-terminal mutations (R862-866A, L908,909V; KA2 $2_{\text {CtermA/B }}$ ) described above. Because we did not observe any dramatic changes in steady-state distribution of KA2 containing the C-terminal mutations $\left(\mathrm{KA} 2_{\mathrm{CtermA} / \mathrm{B}}\right)$ in the immunofluorescence analyses (Fig. 1E,F), we used FACS analysis, a more sensitive quantitative technique. We evaluated KA2 constructs containing mutations in the $\mathrm{C}$ terminus, the intracellular loop, or both, and used FACS analysis to quantitate the amount of cells expressing KA2 on the plasma membrane. Thus, we were able to detect even relatively small changes in surface-expressed KA2. We found that $\sim 4 \%$ of cells exhibited detectable homomeric KA2 surface expression when transfected with the $\mathrm{KA} 2_{\mathrm{CtermA} / \mathrm{B}}$ mutant described by Ren et al. (2003). This represented a 2.4-fold increase in surface expression relative to wildtype KA2 (Fig. 2A). Mutation of the RxR motif alone (KA2 ${ }_{\text {loop }}$ ) did not increase receptor expression on the plasma membrane compared with wild-type KA2; however, the RxR mutation combined with the $\mathrm{C}$-terminal mutations $\left(\mathrm{KA} 2_{\text {loop } / \text { CtermA/B }}\right)$ resulted in the highest surface expression of KA2 (7.5\% of cells). Although this represents a 4.1 -fold increase in expression compared with wild-type KA2 (1.8\%) (Fig. 2 A), the majority of transfected cells did not express KA2 on the plasma membrane regardless of the mutations that were introduced.

To quantitate the effect of oligomerization with GluR6 on KA2 surface expression, we coexpressed GluR6 and KA2 in HeLa cells and analyzed KA2 plasma membrane expression by FACS analysis. We found an increase in KA2 expression on the plasma membrane with GluR6 coexpression; however, surprisingly, this was still a modest increase and represented less than twofold compared with KA2 alone (Fig. 2A). To determine whether the relative DNA concentration in the transfection reaction might regulate the amount of receptor expressed and its oligomerization, we also analyzed the effect of increasing ratios of KA2:GluR6 on surface expression of KA2. We found that increasing the ratios of KA2:GluR6 raised surface expression of KA2 significantly, with

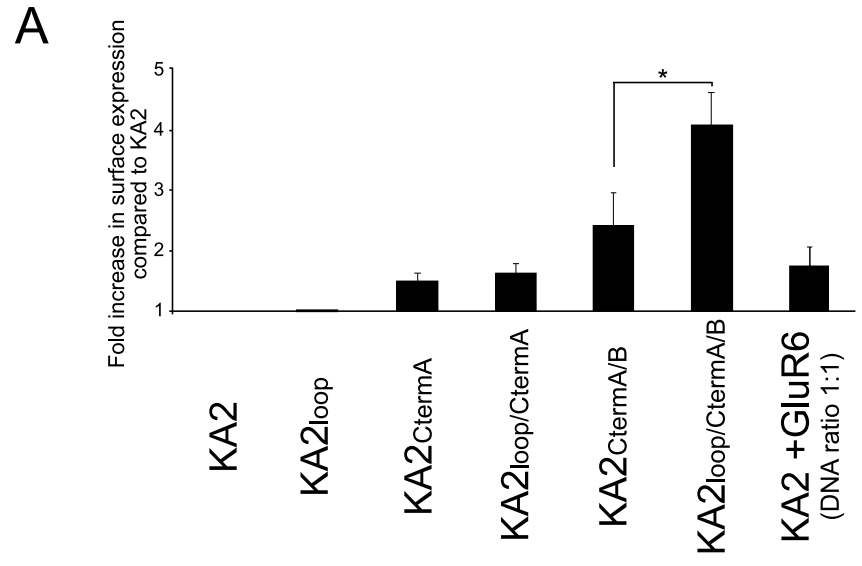

B
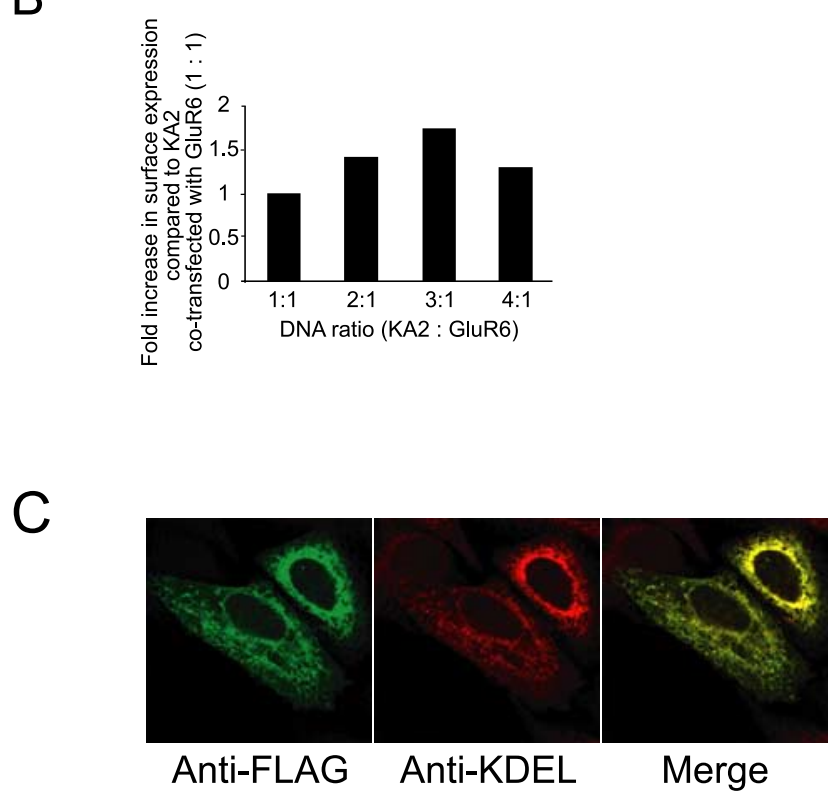

Figure 2. Identification of an arginine-based ER-retention motif ( $R \times R)$ in an intracellular loop of KA2. $A$, FACS analysis of the surface expression of full-length KA2 mutants expressed in HeLa cells. HeLa cells were transfected with FLAG-KA2 wild-type and FLAG-KA2 that contains mutations of putative trafficking motifs within the intracellular loop and the C terminus. Surface expression was analyzed by FACS as described in Materials and Methods. When arginines 580RxR-582 were mutated to alanines in combination with C-terminal $A / B$ mutations, this $\mathrm{KA2} 2_{\text {loop/Cterm } \mathrm{A} / \mathrm{B}}$ mutant showed a fourfold increase in surface expression compared with wildtype KA2. Data represent means \pm SEM of the fold increase in surface expression compared with $K A 2$ based on a percentage of the cells with surface-expressed KA2 in transfected cells $\left[{ }^{*} p<0.05(n=7)\right.$ relative to the surface expression of $\mathrm{KA}_{\mathrm{CtermA}_{\mathrm{B}}}$ mutant; Student's $t$ test]. $\boldsymbol{B}$, Expression of GluR6 affects the surface expression of KA2. GluR6 and FLAG-tagged KA2 were transfected into HeLa cells at different ratios, and the surface expression of KA2 was evaluated by FACS analysis as described in Materials and Methods. C, KA2 is largely ER retained when coexpressed with GluR6 in HeLa cells. FLAG-tagged KA2 and GluR6 were transfected into HeLa cells at a 3:1 ratio. The cells were fixed, permeabilized, and stained with antibodies against FLAG (green) and the ER marker KDEL (red). The merged image shows the extensive colocalization of KA2 with KDEL.

a ratio of 3:1 resulting in a threefold increase compared with KA2 alone in KA2 surface expression (Fig. 2 B). The ability of GluR6 to increase the surface and/or functional expression of KA2 in heterologous cells has been analyzed previously using both biochemical (Hayes et al., 2003; Ren et al., 2003) and electrophysiological techniques (Hayes et al., 2003; Ren et al., 2003), but never quantitatively. Interestingly, even at a 3:1 ratio, the vast majority of KA2 was ER retained (Fig. 2 B). We also used immunofluores- 


\section{A HeLa cells}

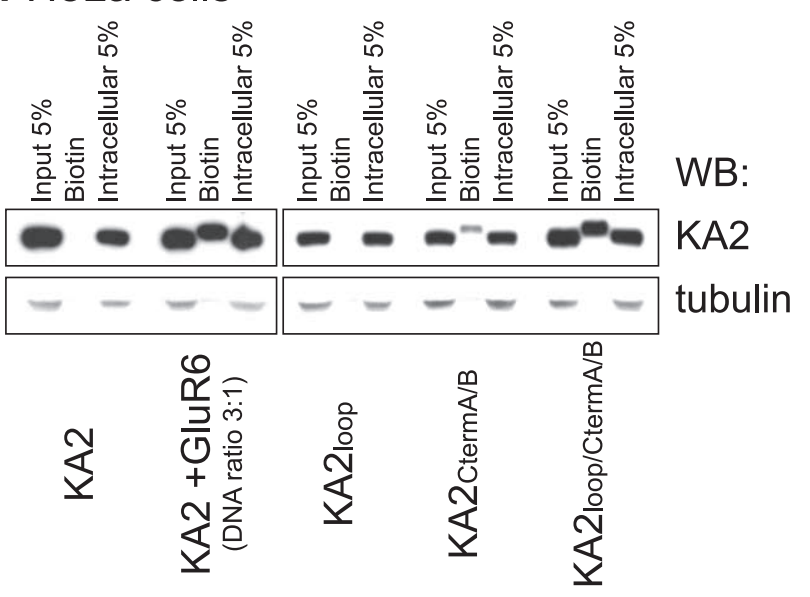

B

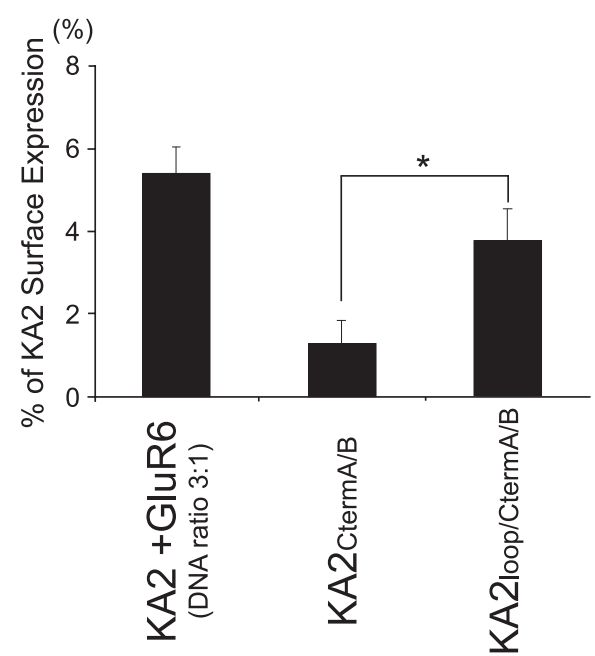

C HEK-293 cells

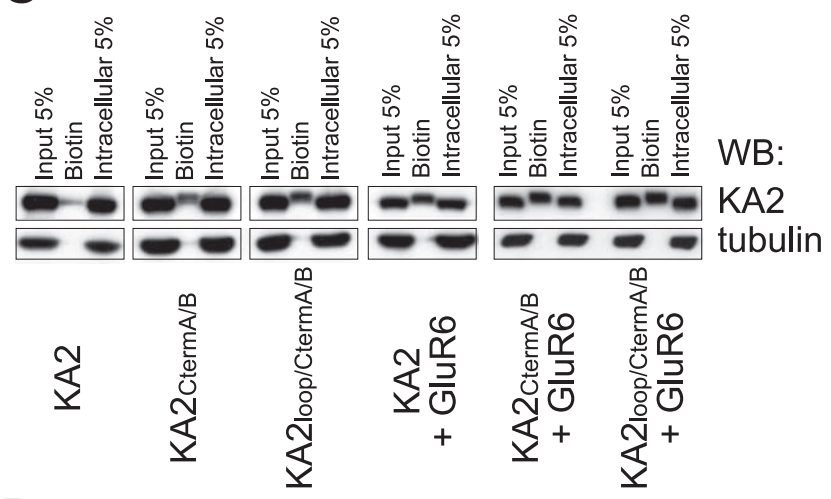

D

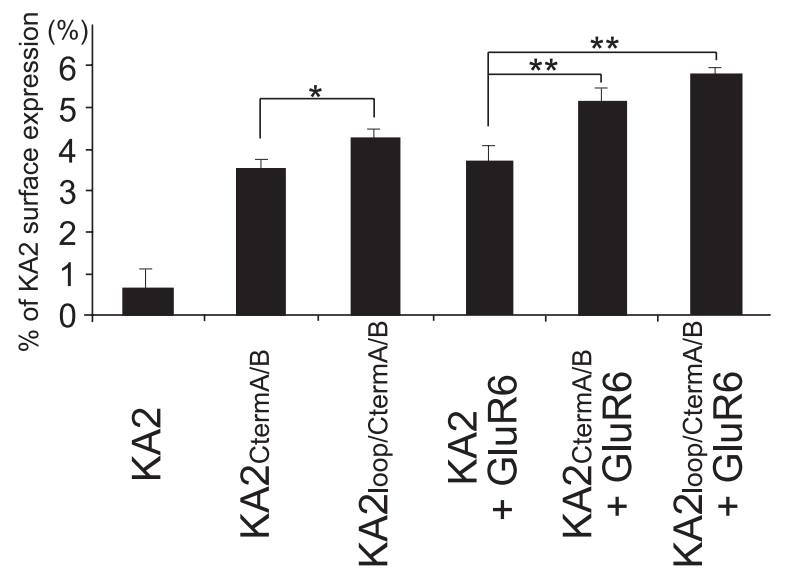

cence microscopy to evaluate the distribution of KA2 coexpressed with GluR6. We transfected HeLa cells with KA2 and GluR6 at a 3:1 ratio and double-labeled the cells with KA2 and KDEL antibodies as described for Figure 1. We found extensive colocalization of KA2 with KDEL (Fig. 2C), even when using a DNA concentration ratio for cotransfections to increase expression of KA2 on the plasma membrane.

In addition to FACS analysis, we used cell surface biotinylation to evaluate the amount of KA2 expressed on the cell surface. Either HeLa cells or HEK-293 cells were transfected with wildtype KA2, KA2 loop $, \mathrm{KA} 2_{\mathrm{CtermA} / \mathrm{B}}, \mathrm{KA} 2_{\text {loop/CtermA/B }}$, alone or with GluR6. Cell surface proteins were biotinylated, and biotinylated proteins were isolated from cell lysates with streptavidinSepharose beads, resolved by SDS-PAGE, transferred to PVDF membranes, and probed by immunoblotting with KA2 antibodies. In HeLa cells, we found that wild-type KA2 was not biotinylated and therefore not surface expressed. A small fraction of $\mathrm{KA} 2_{\mathrm{CtermA} / \mathrm{B}}$, which contains the C-terminal mutations described by Ren et al. (2003), was biotinylated, indicating an increase in surface expression compared with wild-type KA2 (Fig. 3A). The $\mathrm{KA} 2_{\text {loop/CtermA/B }}$ mutant, containing the C-terminal mutations together with the mutation of the ER-retention motif in the intracellular loop, displayed the highest surface expression as revealed by surface biotinylation (Fig. $3 A, B$ ). The intracellular protein tubulin was used as a negative control to confirm that the biotinylation procedure specifically labeled proteins expressed on the plasma membrane. Therefore, in this independent biochemical assay, which monitors the amount of surface-expressed protein in a dish of cells (not the percentage of KA2-expressing cells with KA2 present on the plasma membrane as with FACS analysis), we obtained results consistent with the FACS analyses. Specifically, the biotinylation experiments show that KA2 that contains mutations of the C-terminal trafficking motifs $\left(\mathrm{KA} 2_{\mathrm{CtermA} / \mathrm{B}}\right)$ causes an increase in surface expression relative to wild-type KA2, but that the surface expression of KA2 is most efficient when these $\mathrm{C}$-terminal mutations are combined with the mutation of RxR in the intracellular loop $\left(\mathrm{KA} 2_{\text {loop/CtermA/B }}\right)$.

Because we use HEK-293 cells to express kainate receptors for functional studies, we also evaluated KA2 surface expression in this cell line. Consistent with the results from HeLa cells, the KA2 $2_{\text {loop/CtermA/B }}$ mutant expressed in HEK-293 cells displayed the highest surface expression as revealed by surface biotinylation (Fig. $3 C, D$ ). When the KA2 mutants $\left(\mathrm{KA} 2_{\mathrm{CtermA} / \mathrm{B}}, \mathrm{KA} 2_{\text {loop/CtermA/B }}\right)$ were cotransfected with GluR6, these mutants displayed increased surface expression compared with wild-type KA2 coexpressed with GluR6. Although the KA2 $2_{\text {loop/CtermA/B }}$ mutant cotransfected with GluR6 showed a trend toward increased expression compared with the

$\leftarrow$

Figure 3. Surface expression of KA2 is most robust when the RxR loop mutation is combined with mutations of the $C$-terminal trafficking motifs as revealed using a biotinylation assay. $A, C$, HeLa cells $(\boldsymbol{A})$ or HEK-293 cells $(\boldsymbol{C})$ expressing combinations of wild-type KA2 or mutant KA2 with or without GluR6 were analyzed using the biotinylation assay. The biotinylated surface protein fractions of the whole-cell extracts were separated from the intracellular fractions by conjugation with streptavidin beads and visualized by Western blotting (WB). When arginines 580-RxR-582 within the KA2 intracellular loop were mutated to alanines in combination with C-terminal $A / B$ mutations, this $K A 2_{\text {loop/ctermA } / B}$ mutant was more heavily biotinylated than $K A 2$ containing only the $C$-terminal $A / B$ mutations $\left(K A 2_{\text {cterm } A / B}\right), B, D$, Surface expression of wildtype or mutant KA2 alone or cotransfected with GluR6 expressed in HeLa cells ( $\boldsymbol{B}$ ) and HEK-293 cells $(\boldsymbol{D})$ was quantitated by measuring the band intensity of the biotinylated fraction compared with the intensity of the band representing $5 \%$ of total input using NIH Image software. Error bars indicate SEM. ${ }^{*} p<0.04(n=4$ for $\boldsymbol{B} ; n=3$ for $\boldsymbol{D})$; ${ }^{* *} p<0.01(n=3)$, Student's $t$ test. 
A
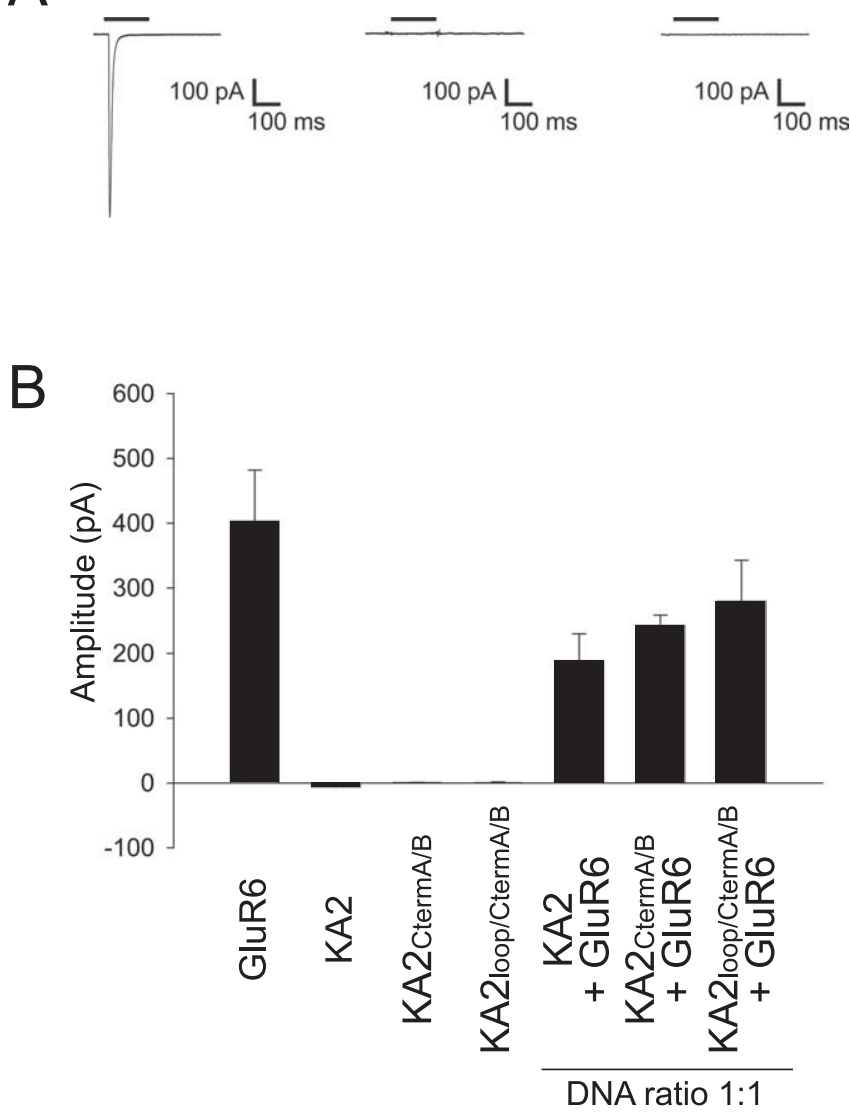

Figure 4. Homomeric wild-type KA2 or KA2 containing C-terminal and loop ER-retention motif mutations do not exhibit kainate-evoked currents in HEK-293 cells. A, Averaged current during whole-cell patch-clamp recordings from HEK-293 cells in response to kainate (300 $\mu \mathrm{m}$; black bar) for cells expressing GluR6 (left), wild-type KA2 (center), or KA2 $2_{\text {loop/ctermA/B }}$ (right) (traces are average of the response to 5-8 consecutive applications of kainate). $\boldsymbol{B}$, Summary data bar graph of mean kainate-evoked current amplitude in HEK-293 cells expressing GluR6 $(n=13), \operatorname{KA2}(n=5), \mathrm{KA}_{\text {CtermA/B }}(n=12), \mathrm{KA}_{\text {loop/CtermA/B }}(n=9), \operatorname{GluR6}$ and KA2 $(n=12)$, GluR6 and KA2 $2_{\text {CtermA/B }}(n=6)$, and GluR6 and KA2 $2_{\text {loop } / \mathrm{CtermA} / \mathrm{B}}(n=6)$. Error bars indicate SEM.

$\mathrm{KA} 2_{\mathrm{CtermA} / \mathrm{B}}$ mutant cotransfected with GluR6, it did not reach significance $(p=0.051 ; n=3)$.

We next evaluated the function of surface-expressed KA2 homomers. The study of Ren et al. (2003) reported that KA2 forms oligomers that are surface expressed but are not functional. However, those findings assumed robust expression of full-length $\mathrm{KA} 2_{\mathrm{CtermA} / \mathrm{B}}$ on the cell surface, but which we find is quite low. Therefore, we re-evaluated whether kainate-evoked currents could be observed in HEK-293 cells expressing KA2 mutants using whole-cell patch-clamp recordings. HEK-293 cells expressing GluR6 alone or GluR6 and KA2 reliably exhibited currents evoked by rapid application of kainate; however, cells expressing wild-type KA2 alone did not exhibit any kainate-evoked current (Fig. 4A, B). Moreover, cells expressing KA2 containing either the C-terminal mutations $\left(\mathrm{KA} 2_{\mathrm{CtermA} / \mathrm{B}}\right)$ or the combined $\mathrm{C}$-terminal and intracellular loop mutations $\left(\mathrm{KA} 2_{\text {loop/CtermA/B }}\right)$ never exhibited kainate-evoked responses (Fig. 4A, B). Coexpression of GluR6 with either of the KA2 mutants $\left(\mathrm{KA} 2_{\mathrm{CtermA} / \mathrm{B}}\right.$ or $\mathrm{KA} 2_{\text {loop/CtermA/B }}$ ) also produced kainate-evoked currents, similar to coexpression of GluR6 and wild-type KA2 (Fig. 4B). Thus, we conclude that, although the combined mutation of the C-terminal and loop ER-retention motifs produces the highest level of surface-expressed homomeric KA2 reported thus far, no func- tional response can be evoked, indicating that homomeric KA2 is not capable of forming a functional ligand-gated ion channel.

The low level of expression of KA2 at the plasma membrane in heterologous cells, when the ER-retention motifs are mutated or in the presence of GluR6, suggests that additional trafficking mechanisms exist in neurons that are not found in heterologous cells. Therefore, we investigated ER-retention and surface expression of endogenous KA2 in brain and in primary neuronal cultures. We first evaluated KA2 trafficking in wild-type brain and compared this to KA2 trafficking in brains from GluR5 $\mathrm{KO}$ or GluR6 KO mice. GluR5 and GluR6 are both kainate receptor subunits capable of oligomerizing with KA2 (Wenthold et al., 1994; Coussen et al., 2002; Gallyas et al., 2003; Hayes et al., 2003; Ren et al., 2003; Coussen et al., 2005) and therefore could potentially influence KA2 trafficking. We used a glycosidase assay (Roche et al., 1999; Hayes et al., 2003) to evaluate whether KA2 was surface expressed or ER retained in mouse brain. We solubilized adult mouse brains collected from wild-type or KO mice, and treated the cell lysates with endo H, PNGase F, or without any enzyme. Proteins were then resolved by SDS-PAGE and immunoblotted with KA2 or GluR6/7 antibodies (Fig. 5A). We found that the majority of KA2 isolated from wild-type brain was endo $\mathrm{H}$ resistant and therefore contained mature N-linked carbohydrates. Endo H resistance indicates that KA2 has traveled beyond the cis-Golgi and is consistent with the majority of KA2 being expressed on the plasma membrane. Both GluR6/7 and KA2 contain mature carbohydrates that are partially endo $\mathrm{H}$ sensitive, resulting in an intermediate shift of mobility with endo $\mathrm{H}$ treatment. The majority of KA2 isolated from GluR5 KO mice was also endo $\mathrm{H}$ resistant and indistinguishable from endogenous KA2 from wild-type brain. In contrast, KA2 from GluR6 KO mice was predominantly endo $\mathrm{H}$ sensitive, resulting in similar migration of KA2 treated with endo H or PNGase F, indicating KA2 is ER retained. In agreement with a previously published report by Christensen et al. (2004), we too observed a substantial reduction in the overall expression of KA2 in GluR6 KO brain. Therefore, these data demonstrate that GluR6, but not GluR5, specifically regulates KA2 trafficking in brain.

To supplement the glycosidase assay, we also cultured primary cortical neurons from wild-type, GluR5 KO, and GluR6 KO mice and analyzed surface expression of KA2. We biotinylated the cultures at 12-14 d in vitro, prepared cell lysates, isolated biotinylated proteins using streptavidin-Sepharose beads, and analyzed surface-expressed proteins by SDS-PAGE and immunoblotting. We found that KA2 was highly expressed on the cell surface in cortical cultures prepared from wild-type and GluR5 KO animals. However, there was a significant reduction in surfaceexpressed KA2 in cortical neurons prepared from GluR6 KO mice (Fig. $5 B$ ). These data confirm our glycosidase results from adult brain demonstrating that GluR6 regulates KA2 trafficking and surface expression. Moreover, these data show that, in neurons, GluR6 is required for efficient surface expression of KA2, in contrast to non-neuronal heterologous cells, indicating that critical mechanisms regulating the trafficking of KA2 exist in neurons that are not present in heterologous cells.

\section{Discussion}

ER-retention and retrieval motifs are dominant trafficking signals that are often encoded in individual subunits of multiprotein complexes, thereby providing a quality control mechanism to ensure proper oligomerization (Ellgaard and Helenius, 2003; Trombetta and Parodi, 2003). The best-characterized ERretention motifs are the KDEL sequence for soluble ER proteins, 
A
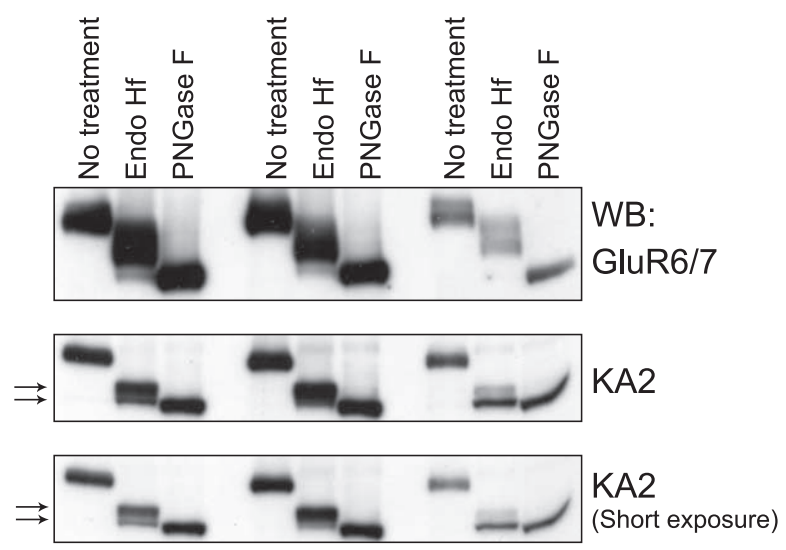

Wild-type GluR5 KO GluR6 KO

B
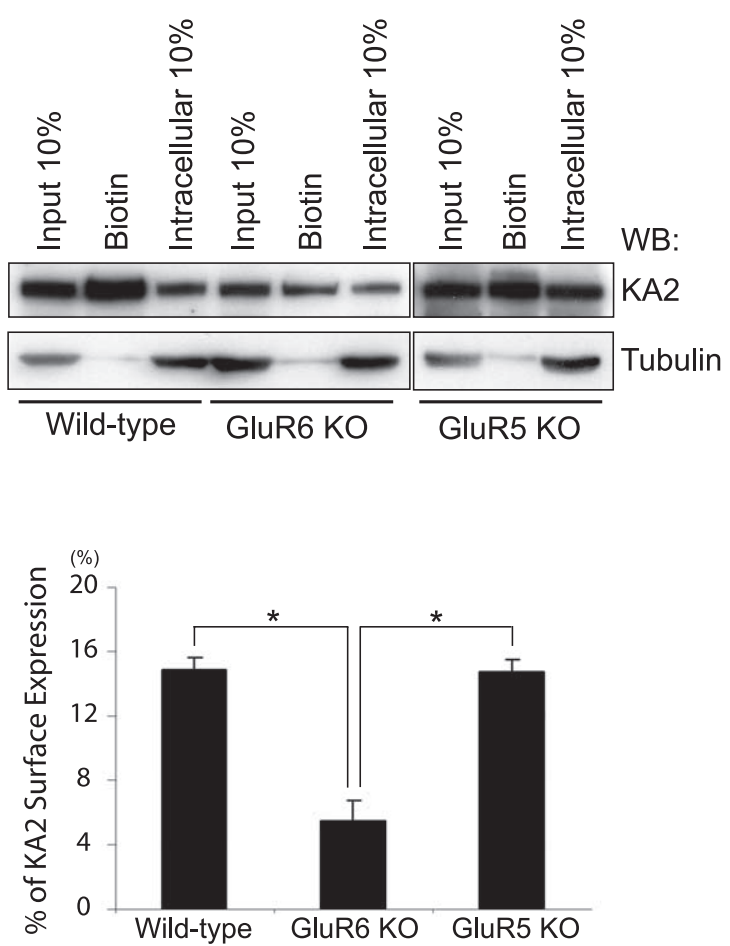

Figure 5. KA2 does not traffic to the cell surface efficiently in neurons lacking GluR6. $A$, The majority of $\mathrm{KA} 2$ in $\mathrm{GluR6} \mathrm{KO}$ brain is ER retained. The whole-brain lysate $(50 \mu \mathrm{g})$ from wild-type, GluR5 K0, and GluR6 KO mouse was treated with endo $\mathrm{H}$ or PNGase $\mathrm{F}$, and immunoblots were probed with GluR6/7 or KA2 antibodies. Mature forms of both GluR6 and KA2 display partial endo $\mathrm{H}$ sensitivity. $\boldsymbol{B}$, Less $\mathrm{KA} 2$ is expressed on the cell surface in primary cultured GluR6 KO cortical neurons than in wild-type or GluR5 K0 cortical neurons. Primary cultures of cortical neurons were obtained from $\mathrm{P} 0-\mathrm{P} 1$ pups. The neurons were maintained for 2 weeks and surface-biotinylated with sulfo-NHS-LC-biotin. The biotinylated surface protein fractions of the whole-cell extracts were separated from the intracellular fractions by conjugation with streptavidin beads, resolved by SDS-PAGE, and probed with KA2 or tubulin antibodies. Surface expression of KA2 was quantitated by measuring the band intensity of biotinylated fraction compared with the intensity of the band representing $10 \%$ of total input using NIH Image software. Error bars indicate SEM. ${ }^{*} p<0.01$ ( $n=5$ for wild-type and GluR6 K0; $n=3$ for GluR5 K0), Student's $t$ test. WB, Western blot. the KK motif for type I integral membrane proteins, and the RxR motif for multimembrane spanning integral membrane proteins (Ma and Jan, 2002; Ellgaard and Helenius, 2003). Several ion channels (e.g., ATP-sensitive potassium channels) and receptors (e.g., NMDA receptors) contain RxR motifs that regulate surface expression of heteromeric complexes (Ma and Jan, 2002). For example, NR1-1, the most prevalent splice variant of the NR1 subunit of NMDA receptors, contains an RxR motif that regulates surface expression (Standley et al., 2000; Scott et al., 2001). NR1-1 requires oligomerization with NR2 subunits before exiting the ER and trafficking to excitatory synapses. Therefore, NR1 is rarely, if ever, expressed as a homomeric channel in neurons. It is likely that NR1 homomers are excluded because NR1 contains the glycine binding site, whereas NR2 subunits contain glutamate binding sites. Thus, NMDA receptor hetero-oligomerization promotes the surface expression of NMDA receptors containing both glycine and glutamate binding sites. Because binding of agonists to both of these sites is required for channel opening, ERretention of homomeric assemblies of NMDA receptors ensures that only functional heteromeric receptors are expressed at the plasma membrane.

Based on the trafficking of NMDA receptors, it seemed likely that other subtypes of glutamate receptors might use similar checkpoints to exclude homomeric receptor complexes from trafficking to the plasma membrane. Endogenous kainate receptors are often heteromers consisting of complexes of GluR6 and KA2 subunits (Wenthold et al., 1994). KA2 is ER retained unless coexpressed with other kainate receptor subunit (GluR5-7). In the current study, we have characterized ER-retention of the KA2 subunit in neurons or KA2 expressed in heterologous cells. KA2 contains a stretch of arginines (862-RRRRR-866) in its C terminus that appears to be a candidate for an arginine-rich motif necessary for ER localization, and a recent publication reported that this sequence regulated ER-retention of KA2 (Ren et al., 2003). This study relied heavily on the analysis of chimeras of the plasma membrane reporter molecule Tac and the cytosolic C terminus of KA2 (amino acids 825-979). In our own laboratory, we find similar effects on the surface expression of Tac-KA2 when the C-terminal trafficking motifs are mutated (Fig. $1 B-D$ ) as those observed by Ren et al. (2003). However, we find that when this mutation (862-RRRRR-866) is introduced in the context of full-length KA2, these mutations have very little effect on ERretention. All previously identified trafficking motifs that regulate glutamate receptor localization are contained within the cytosolic C-terminal domains of glutamate receptor subunits (Wenthold et al., 2003; Isaac et al., 2004; Vandenberghe and Bredt, 2004; Jaskolski et al., 2005). In contrast, our studies reveal a novel RxR motif (580-RAR-582) within an intracellular loop of KA2, a region previously overlooked in receptor trafficking of kainate receptors and other glutamate receptor subtypes. Therefore, this novel KA2 motif represents a departure from the NMDA model for receptor trafficking.

It should be noted that mutation of the C-terminal (862RRRRR-866) motif and the loop RxR (580-RAR-582) motif have a negligible effect on KA2 surface expression when mutated individually or together, unless the C-terminal LL mutation (908-LL909) is also introduced. The reason for this phenomenon remains unclear. The dileucine motif was originally identified as an endocytic motif (Ren et al., 2003); however, the original study provided no evidence of the LL regulating endocytosis. Whereas disruption of receptor endocytosis could certainly lead to increased expression on the plasma membrane, this would not necessarily release receptor from retention in the ER. Although we find no 
direct evidence that KA2 undergoes endocytosis or resides in endosomes, all of our analyses demonstrate that the LL mutation is critical for increasing surface expression.

Our data and those of others (Ren et al., 2003) demonstrate that KA2 contains cis-acting trafficking motifs that regulate surface expression. However, we find that even when the C-terminal trafficking motifs (862-RRRRR-866, 908-LL-909) and the RxR motif in the intracellular loop (580-RAR-582) are mutated, KA2 is still predominantly localized to the ER. Interestingly, KA2 that contains mutations in all of the described trafficking motifs displays little expression on the plasma membrane compared with GluR6, which forms functional homomeric channels and is efficiently expressed on the plasma membrane. Therefore, it is possible that KA2 uses additional mechanisms beyond the classic ER-retention motifs to regulate its surface expression.

In this study, we have analyzed, for the first time, the trafficking of endogenous KA2 in wild-type brain versus kainate receptor subunit $\mathrm{KO}$ brain and compared this to the trafficking of KA2 expressed in heterologous cells. Our findings demonstrate that oligomerization of KA2 with additional kainate receptor subunits, specifically GluR6, is critical for surface expression in vivo. We find that KA2 is predominantly ER retained in GluR6 KO mice, but is not ER retained in wild-type mice or in GluR5 KO mice, demonstrating that GluR6 specifically regulates KA2 surface expression. Whether oligomerization with GluR6 is essential to mask ER-retention motifs in KA2 is unclear. Our quantitative analyses of the trafficking of GluR6/KA2 heteromers in heterologous cells, in which we find only $5.6 \%$ of KA2 is surface expressed on coexpression with GluR6, suggests that oligomerization of kainate receptors may not be the entire story. Whereas oligomerization may be important for masking ER-retention motifs, it is also possible that oligomerization plays a more general role in protein folding of KA2 that greatly facilitates egress from the ER. Given the dramatic differences in KA2 trafficking in neurons and in heterologous cells, it is likely that neuron-specific factors contribute to the efficient expression of KA2 on the plasma membrane in vivo.

In summary, we identified novel mechanisms regulating the surface expression of KA2. These mechanisms involve ERretention motifs that prevent plasma membrane expression of KA2 homomers and in neurons hetero-oligomerization specifically with GluR6. The regulation of kainate receptor expression via ER-retention prevents surface expression of the nonfunctional KA2 homomers, and specifically promotes plasma membrane expression of heteromeric receptors containing GluR6 and $\mathrm{KA} 2$, the most commonly observed kainate receptor subunit composition in the brain. Thus, the surface expression of kainate receptors, like NMDA receptors, is regulated by a combination of ER-retention of individual subunits and hetero-oligomerization to achieve a high probability of functional heteromeric complexes expressed at excitatory synapses.

\section{References}

Bahn S, Volk B, Wisden W (1994) Kainate receptor gene expression in the developing rat brain. J Neurosci 14:5525-5547.
Christensen JK, Paternain AV, Selak S, Ahring PK, Lerma J (2004) A mosaic of functional kainate receptors in hippocampal interneurons. J Neurosci 24:8986-8993.

Coussen F, Normand E, Marchal C, Costet P, Choquet D, Lambert M, Mege RM, Mulle C (2002) Recruitment of the kainate receptor subunit glutamate receptor 6 by cadherin/catenin complexes. J Neurosci 22:6426-6436.

Coussen F, Perrais D, Jaskolski F, Sachidhanandam S, Normand E, Bockaert J, Marin P, Mulle C (2005) Co-assembly of two GluR6 kainate receptor splice variants within a functional protein complex. Neuron 47:555-566.

Ellgaard L, Helenius A (2003) Quality control in the endoplasmic reticulum. Nat Rev Mol Cell Biol 4:181-191.

Gallyas Jr F, Ball SM, Molnar E (2003) Assembly and cell surface expression of KA-2 subunit-containing kainate receptors. J Neurochem 86:1414-1427.

Hayes DM, Braud S, Hurtado DE, McCallum J, Standley S, Isaac JT, Roche KW (2003) Trafficking and surface expression of the glutamate receptor subunit, KA2. Biochem Biophys Res Commun 310:8-13.

Isaac JT, Mellor J, Hurtado D, Roche KW (2004) Kainate receptor trafficking: physiological roles and molecular mechanisms. Pharmacol Ther 104:163-172.

Jaskolski F, Coussen F, Mulle C (2005) Subcellular localization and trafficking of kainate receptors. Trends Pharmacol Sci 26:20-26.

Lerma J (2003) Roles and rules of kainate receptors in synaptic transmission. Nat Rev Neurosci 4:481-495.

Ma D, Jan LY (2002) ER transport signals and trafficking of potassium channels and receptors. Curr Opin Neurobiol 12:287-292.

Margeta-Mitrovic M, Jan YN, Jan LY (2000) A trafficking checkpoint controls $\mathrm{GABA}_{\mathrm{B}}$ receptor heterodimerization. Neuron 27:97-106.

Porter RH, Eastwood SL, Harrison PJ (1997) Distribution of kainate receptor subunit mRNAs in human hippocampus, neocortex and cerebellum, and bilateral reduction of hippocampal GluR6 and KA2 transcripts in schizophrenia. Brain Res 751:217-231.

Ren Z, Riley NJ, Garcia EP, Sanders JM, Swanson GT, Marshall J (2003) Multiple trafficking signals regulate kainate receptor KA2 subunit surface expression. J Neurosci 23:6608-6616.

Roche KW, Tu JC, Petralia RS, Xiao B, Wenthold RJ, Worley PF (1999) Homer $1 \mathrm{~b}$ regulates the trafficking of group I metabotropic glutamate receptors. J Biol Chem 274:25953-25957.

Scott DB, Blanpied TA, Swanson GT, Zhang C, Ehlers MD (2001) An NMDA receptor ER-retention signal regulated by phosphorylation and alternative splicing. J Neurosci 21:3063-3072.

Standley S, Roche KW, McCallum J, Sans N, Wenthold RJ (2000) PDZ domain suppression of an ER-retention signal in NMDA receptor NR1 splice variants. Neuron 28:887-898.

Trombetta ES, Parodi AJ (2003) Quality control and protein folding in the secretory pathway. Annu Rev Cell Dev Biol 19:649-676.

Vandenberghe W, Bredt DS (2004) Early events in glutamate receptor trafficking. Curr Opin Cell Biol 16:134-139.

Wenthold RJ, Trumpy VA, Zhu WS, Petralia RS (1994) Biochemical and assembly properties of GluR6 and KA2, two members of the kainate receptor family, determined with subunit-specific antibodies. J Biol Chem 269:1332-1339.

Wenthold RJ, Prybylowski K, Standley S, Sans N, Petralia RS (2003) Trafficking of NMDA receptors. Annu Rev Pharmacol Toxicol 43:335-358.

Wisden W, Seeburg PH (1993) A complex mosaic of high-affinity kainate receptors in rat brain. J Neurosci 13:3582-3598.

Zerangue N, Schwappach B, Jan YN, Jan LY (1999) A new ER trafficking signal regulates the subunit stoichiometry of plasma membrane K(ATP) channels. Neuron 22:537-548. 